\title{
SPANNING HYPERFOREST OF A HYPERGRAPH
}

\author{
THINH D. NGUYEN
}

\begin{abstract}
We are interested in the complexity of a problem involving spanning hyperforests (a union of hypertrees, which covers all of the vertices) of a $k$-hypergraph. We describe the relevant definitions for hypergraphs and our problem in details.
\end{abstract}

\section{Notations And PRoblem StATEMEnt}

First, it is convenient to delineate our problem on hypergraph as follows.

Definition 1. (SPANNING HYPERFOREST ROOT SET):

For a directed hypergraph $D$ and an integer $k \geq 1$, determine whether there exists a spanning hyperforest for $D$ which has a root-set of size at most $k$.

To fully understand the above definition, some remarks are necessary.

- It is not difficult to show that SPANNING HYPERFOREST ROOT SET is in NP: in particular, if a root-set of the suitable size is provided, then a spanning hyperforest with that root-set can be found in polynomial time.

- It is also trivial to find a value of $k$ for which $(D, k)$ is a YES instance: for instance $k=|V(D)|$ (in which case the empty hypergraph is a spanning hyperforest with root set $k$ ).

- Considered as an optimisation problem, it is usually easy to find values $k<|V(D)|$ for which $(D, k)$ remain Yes instances, though it is not clear how easily one can find the optimum.

1.1. Symmetric variant definition. A natural question is whether this is true in the special case where the input hypergraph is symmetric, in the sense that for any edge $e=(t(e), h(e))$ and for any $v \in s(e):=t(e) \cup h(e)$, there is also an edge $e^{\prime}=(s(e) \backslash\{v\}, v)$. In the following, we will prove the hardness of this variant. So, a formal definition is in need.

Definition 2. (SPANNING SYMMETRIC HYPERFOREST ROOT SET):

Given an undirected hypergraph $G$ and a number $k$, determine whether there is a spanning hyperforest with root set of size at most $k$ in the symmetric directed hypergraph $D_{G}$.

We will reduce from MAXIMUM UNIQUELY RESTRICTED BIPARTITE MATCHING (MURBM) problem which definition is given right below.

\section{Definition 3. (MAXIMUM UNIQUELY RESTRICTED BIPARTITE MATCH- ING):}

Key words and phrases. hypergraph, hypertree, optimization, root, spanning.

Perebor. 
Before really defining MURBM, a subdefinition is really of help. A uniquely restricted matching in a graph $B$ is a matching $M$ such that the induced subgraph of $B$ over the vertices of $M$ has exactly one perfect matching ( $M$ itself).

Now, the MURBM problem asks for a given bipartite graph $B$ and a given number $n$ whether there exist a uniquely restricted matching in the graph with at least $n$ edges.

Also note that a matching is uniquely restricted if and only if there exists no cycle in the graph whose edges alternate between being in the matching and not being in the matching. (This is easy to show, so we will not be include the proof here).

1.2. The claim and some auxiliary definitions. Having all required definition defined and explained, we are now ready to state the main claim of this paper.

Claim 4. We have that MURBM $\leq_{p}$ SSHRS

In this claim, the hardness of MURBM is known since [4]. Now, some relevant definitions are useful.

In the following, we are broadly following the definitions of Cambini, Gallo, and Scutella [5].

- A hypergraph is a pair $G=(V, E)$, where $E \subseteq \mathcal{P}(V)$. If each $e \in E$ has the same cardinality $k$, we call $G$ a $k$-uniform hypergraph (or $k$-hypergraph).

- A directed hypergraph is a pair $D=(V, E)$, where in our setting we let $E \subseteq \mathcal{P}(V) \times V$ be the set of hyper-edges. For each edge $e \in E$, we let $t(e)=\pi_{1}(e) \subseteq V$ be the "tail" of the edge, and $h(e)=\pi_{2}(e) \in V \backslash t(e)$ be the "head" of the edge. Thus we consider hypergraphs where each edge has exactly one head (more general definitions are common).

- We may associate a "symmetric" directed hypergraph $D_{G}=\left(V, E^{\prime}\right)$ of this sort to any hypergraph $G=(V, E)$, by replacing each undirected edge $e \in E$ with a collection of directed variants $E_{e}^{\prime}=\{(e \backslash v, v) \mid v \in e\}$ and letting $E^{\prime}=\bigcup_{e \in E} E_{e}^{\prime}$.

- A directed cycle in a directed hypergraph $D=(V, E)$ is just a vertex sequence $\left(v_{0}, v_{1}, \ldots, v_{\ell}\right)$ for which $v_{i+1} \neq v_{i}$ for $0 \leq i<\ell, v_{0}=v_{\ell}$, and for which for each $0 \leq i<\ell$ there is an edge $e_{\ell}$ for which $v_{i} \in t\left(e_{i}\right)$ and $v_{i+1}=h\left(e_{i}\right)$.

- A hyperforest is a directed hypergraph in which all vertices have in-degree either zero or one, and which has no cycles in the above sense. (Note that we are diverging here from the terminology in our reference above, which does not explicitly consider whether the hypergraph is connected; but this cannot be taken for granted in our setting.) The set of nodes of in-degree zero we call the "root set" of the hyperforest.

- A spanning hyperforest $T$ for a directed hypergraph $D=(V, E)$, is simply a subgraph $T \subseteq D$ of the hypergraph which contains all of the vertices $V$ and which is a hyperforest.

\section{REDUCING MURBM to SSHRS}

2.1. Description of the reduction. The reduction from MURBM to SSHRS is simple: we are given a MURBM instance consisting of bipartite graph $B$ and number $n$. Suppose $B$ has vertex parts $V_{1}$ and $V_{2}$. Define $N(v)$ to be the set of 
neighbors of $v$ in $B$. Then define a hypergraph $G=(V, E)$ with vertices $V=V_{1}$ and edges $E=\left\{N(v) \mid v \in V_{2}\right\}$. Next, define $k=\left|V_{1}\right|-n$. In other words, construct the hypergraph $G$ whose bipartite graph model is $B$. Output $G$ and $k$ as the SSHRS instance output of the reduction.

2.2. Correctness of the above construction. The logic behind the reduction is described in the following proof.

Proof. Choosing a spanning hyperforest in $D_{G}$ is equivalent to choosing a set of edges in $D_{G}$ subject to certain constraints. But choosing a set of edges in $D_{G}$ (subject to certain constraints) is equivalent to choosing a set of edges in $G$ and choosing a vertex to be the head from each (subject to certain constraints). Since a hyperforest allows no cycles, the same edge of $G$ directed in different ways cannot be used (as two such "duplicate" edges form a cycle). Since in a hyperforest the vertices have max in-degree one, the same vertex cannot be chosen as the head of multiple different edges. In other words, the choice of edges in $G$ and heads of those edges that comprises a choice of spanning hyperforest is equivalent to the choice of a matching in the bipartite graph model of $G$ (subject to certain constraints). But by our construction, the bipartite graph model of $G$ is exactly the bipartite graph $B$, so choosing a spanning hyperforest in $D_{G}$ is equivalent to choosing a matching in $B$ subject to certain constraints.

One may ask what exactly those constraints are. A hyperforest cannot have any cycles, and must have vertex in-degrees of at most 1 . The vertex in-degree constraint is already met by any matching, so the only left constraint is to have no cycles. But a cycle in the hyperforest corresponds to a cycle in the bipartite graph model of $G$ such that every second edge is in the corresponding "matching". Thus, the cycle-free condition of the hyperforest is equivalent to the condition that there exists no cycle in the graph whose edges alternate between being in the matching and not being in there.

Thus, hyperforests in $G$ correspond to uniquely restricted matchings in $B$. All that is left is to show that the parameters also match: that having a hyperforest with root set size at least $k$ corresponds to having a matching of size at least $n$. Every vertex in a spanning hyperforest has in-degree either 0 or 1, so the number of in-degree- 1 vertices is equal to the total in-degree in the spanning hyperforest. But the total in-degree is also equal to the number of edges since each edge has exactly one head. Thus, the number of in-degree- 1 vertices in the spanning hyperforest is equal to the number of edges. We also know that the size of the matching equals the number of edges in the hyperforest. Thus the size of the matching equals the number of in-degree- 1 vertices in the hyperforest. Furthermore, the number of roots is by definition the number of in-degree- 0 vertices. Thus, the size of the root set plus the size of the matching is equal to the number of vertices $|V|=\left|V_{1}\right|$. Therefore, the hyperforest has root set size at most $k=\left|V_{1}\right|-n$ if and only if the matching has size at least $n$.

\section{Conclusion}

Garey and Johnson [1] shapes their theory based on previous primal works of Cook, Levin and Karp. Johnson [2] moves on with the guide to this theory. As long as we study a mathematical conjecture, we should encourage ourselves of having enough labouring hours on popular maths books like these. Then, reading some 
articles on theory of computing like [3] is a good practice. Only after that, could we think of the ultimate final for all mathematics sciences.

\section{REFERENCES}

1. Michael R. Garey, David S. Johnson, Computers and Intractability: A Guide to the Theory of NP-Completeness

2. David S. Johnson, The NP-Completeness Column: An Ongoing Guide. pp.393-405

3. Phan Dinh Dieu, Le Cong Thanh, Le Tuan Hoa, Average Polyno-mial Time Complexity of Some NP-Complete Problems, Theor. Comput. Sci. 46(3): pp.219-237 (1986)

4. M. C. GolumbicT. HirstM. Lewenstein, Uniquely Restricted Matchings, Algorithmica, vol. 31.2 , pp. 139-154, 2001

5. Riccardo Cambini, Giorgio Gallo, Maria G. Scutella, Flows on hypergraphs, Mathematical Programming, Vol. 78.2, pp.195-217, 1997

Current address: Department of Mathematics, Moscow State University

Email address: kosmofarmer@yandex.com 